\title{
Two-dimensional Ising model with Einstein site phonons
}

\author{
L. Pili ${ }^{1}$ and S. A. Grigera ${ }^{1,2}$ \\ ${ }^{1}$ Instituto de Física de Líquidos y Sistemas Biológicos, UNLP-CONICET, La Plata 1900, Argentina \\ ${ }^{2}$ SUPA, School of Physics and Astronomy, University of St Andrews, North Haugh, St Andrews KY16 9SS, United Kingdom
}

(Received 1 November 2018; published 23 April 2019)

\begin{abstract}
We consider a simple Ising magnetic model in two dimensions with Einstein site phonons and study it using Monte Carlo simulations that take into account both degrees of freedom simultaneously. In nonfrustrated systems, like the square lattice with ferromagnetic and antiferromagnetic interactions, we find that the coupling of the magnetic to the elastic degrees of freedom gradually lowers the magnetic ordering transition until it is completely suppressed at a critical value of the coupling constant. Above this the system suffers a simultaneous magnetic and structural transition into a dimerized state with lower crystalline symmetry and ferromagnetic clusters antiferromagnetically aligned. In the case of the Kagomé lattice with antiferromagnetic interactions, which is frustrated, we show that a similar ordered state takes place when the coupling constant is above a critical value.
\end{abstract}

DOI: 10.1103/PhysRevB.99.144421

\section{INTRODUCTION}

In every real magnetic material there is an interplay between the magnetic and elastic degrees of freedom. While in many cases this is of no consequence for the magnetic order, and can be neglected, there are a growing number of cases where this interplay is key to understanding the ground state and excitations of magnetic materials. Starting with the theoretical prediction of the so-called "spin-Peierls" effect [1], a progressive spin-lattice dimerization occurring at low temperatures, observed experimentally in $\mathrm{CuGeO}_{3}$ [2], there has been a rapidly growing literature that addresses this issue both from theoretical [3-10] and experimental [11-18] perspectives.

Theoretical models have considered classical Ising and Heisenberg models coupled with global Debye distortions (e.g., Ref. [3] or Ref. [10]), unconstrained (the bond model of Ref. [6]) and Einstein site phonons (e.g., Ref. [8] or Ref. [9]) which are a good approximation for systems dominated by optical phonons. Treating both degrees of freedom simultaneously can be a daunting task. Some simulations exist in the literature $[3,7,10]$, but the usual method is to perform a Gaussian integral over the set of displacement coordinates in the partition function. The phonons are then integrated out to obtain an effective spin Hamiltonian which redresses the exchange constants and can introduce additional interaction terms (see, e.g., Ref. [8]). Analytical work, or simulations, are then performed on the effective system.

The two-dimensional (2D) Ising model is probably the simplest magnetic model to show a nontrivial phase transition, and is among the most studied models. Surprisingly there is no work in the literature that describes the case of the 2DIsing model under Einstein distortions. In this work we do a full Monte Carlo simulation, treating simultaneously spin and elastic degrees of freedom, of the classical Ising model on the square and Kagomé lattices. Following Ref. [8] we consider
Einstein distortions and a linear coupling between both degrees of freedom. The model and the Monte Carlo algorithm used for the simulations and the consistency checks performed on our system are discussed in Sec. II. A conception usually found in the literature is that distortions are important only in frustrated systems and that their main effect is to help ordering by relief of frustration $[8,9,19-21]$. Instead, we find that in the unfrustrated square lattice (Sec. III A) this coupling weakens the magnetic ordering transition into the fully polarized state. Upon increase above a critical value the coupling leads to a structural distortion simultaneous with ordering into a different magnetic state, which we label a checkerboard phase, or $\mathrm{CB}$. This $\mathrm{CB}$ phase is a zero-magnetization state, composed of ferromagnetic clusters ordered antiferromagnetically with respect to each other. In the frustrated Kagomé case (Sec. III B) we find above a critical coupling an ordered state which shares many similarities with the $\mathrm{CB}$ phase.

\section{MODEL AND METHODS}

For our study we use a simple model that takes into account the coupling between magnetic and elastic degrees of freedom the so-called Einstein site phonon spin model [8]. In this model the sites have independent displacements given by a set of independent harmonic oscillators. Here one is assuming that the most important lattice distortion contribution is coming from optical phonons. This is a reasonable assumption given that in real materials the active magnetic lattice is usually a sublattice of a more complex crystal structure (e.g., the pyrochlore Dy lattice in $\mathrm{Dy}_{2} \mathrm{Ti}_{2} \mathrm{O}_{7}$ ). The Hamiltonian is then given by

$$
\mathcal{H} /\left|J_{0}\right|=\sum_{\langle i, j\rangle} J\left(r_{i j}\right) S_{i} S_{j}+\frac{K_{\mathrm{e}}}{2} \sum_{i}\left|\mathbf{u}_{i}\right|^{2} .
$$


Here $J_{0}$ gives the energy scale, $J\left(r_{i j}\right)$ is the exchange constant in units of $J_{0}$ that depends on the relative position of the sites $i$ and $j, S_{i}$ are Ising variables, and the sum $\langle i, j\rangle$ is over nearest neighbors only. The $\mathbf{u}_{i}$ are the dimensionless displacements for each site $i$ in units of the undistorted nearest neighbors distance $a$ and $K_{\mathrm{e}}$ is a dimensionless elastic constant. If we consider the displacements to be sufficiently small compared with the lattice parameter $\left(\left|u_{i}\right| \ll a\right)$ then it is reasonable to expand $J_{i j}$ to a linear dependence on the relative site positions $r_{i j}$ :

$$
J\left(r_{i j}\right)=\operatorname{sgn}\left(J_{0}\right)\left[1-\alpha\left(r_{i j}-1\right)\right],
$$

where $\alpha$ is a dimensionless coupling constant. We will use $\alpha$ as a control parameter to measure the degree of coupling to lattice distortions.

To simulate the elastic distortions we consider polar coordinates, $\theta$ is treated like a clock model of 360 equally spaced angles and the displacement $\rho$ is chosen randomly in a distribution from 0 to a temperature dependent maximum $\delta_{\max }(T)$. The use of the latter has no impact on the results obtained from the simulation, it is introduced merely as a way to optimize the speed of the simulations by avoiding the proposal of extremely unlikely moves at low temperatures. To determine $\delta_{\max }(T)$ we simulate a spinless lattice with a large $\delta_{\max }$ and calculate a histogram of the displacements at several temperatures. From each of these we choose a $\delta_{\max }(T)$ such that it includes $80 \%$ of the histogram. We then fit a power law to these points and use the fitted function in the simulations. The function that fits best has, as expected, a square root dependence in $T / J_{0}$. A spinless simulation of a square lattice using this algorithm gives the correct specific heat $\left(C_{v}=1\right)$ and the correct temperature dependence for the mean square displacement $\left(\left\langle u^{2}\right\rangle \propto T\right)$.

In our Monte Carlo simulation we treat simultaneously the magnetic and elastic degrees of freedom using a BornOppenheimer (BO) approximation, that is, by assuming that the relaxation times of the magnetic degrees are much shorter than the elastic. Each step of the simulation is split into elastic and magnetic moves. The $\mathrm{BO}$ approximation translates into the fact that each elastic move is done with a relaxed magnetic configuration. The algorithm proceeds as follows:

We do $P$ elastic Monte Carlo steps (MCS), each of which consists of the following:

(1) Choose a random site.

(2) Propose a move by picking at random an angle and a displacement (from 0 to $\delta_{\max }$ ).

(3) Calculate the exchange constants for the proposed spacial configuration.

(4) Calculate the total energy of the system (magnetic+elastic) and accept or reject the move according to Metropolis.

(5) Make $Q$ magnetic moves, each move consists of:

(a) Flip one spin at random

(b) Calculate the change in magnetic energy

(c) Accept or reject according to Metropolis

(d) Repeat (a) to (c) until each spin has been chosen at least once on average.

(6) Repeat 1 to 5 until each site has been chosen at least once on average.

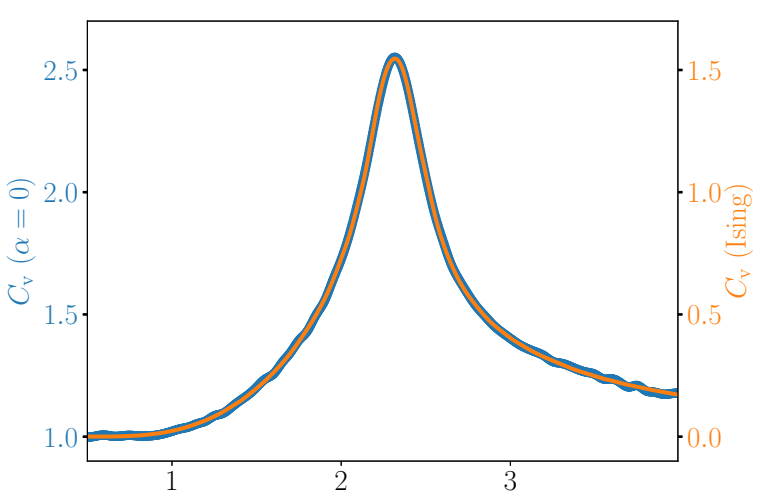

FIG. 1. Comparison between the specific heat of a decoupled elastic system (blue line, left axis) and a static Ising system (orange line, right axis). As expected the specific heat of the former is a simple sum of the elastic contribution $\left(C_{v}^{\mathrm{el}}=1\right)$ and the magnetic Ising part (the right axis is shifted accordingly in the plot).

We have checked that our results are independent of the precise choice of the ratio $P / Q$ by running different simulations on lattices with $N$ sites with $Q$ varying from 1 to 300 , that is, the number of moves for each $Q$ being from $N$ to $300 N$ times those for each $P$. We have used square lattices with $L$ from 4 to 24 and typically with $P=10^{7}$ MCS. Quantities are averaged over time after a waiting period of $P=50000 \mathrm{MCS}$ to allow for equilibration (see [22]). The figures in this paper are all for $L=16$.

The energy scales for magnetic and elastic degrees of freedom can be characterized by the critical temperature of the decoupled Ising system $T_{c}^{0}$ and the melting temperature $T^{*}$. The latter can be defined in our system by means of the Lindemann criterion in two dimensions $[23]\left(\sqrt{\left\langle u^{2}\right\rangle} \approx 0.1\right)$, and the former can be determined by simulating the decoupled magnetic system. Using equipartition one gets $T^{*} \approx$ $\left|J_{0}\right| K_{\mathrm{e}} / 200$. In order to work in the limit $|u| \ll 1$ one must choose $K_{\mathrm{e}}$ such that $T_{c}^{0} / T^{*} \ll 1$. For the simulations of this work we have chosen $K_{\mathrm{el}}=7200$ which means $T^{*} / T_{c}^{0} \approx 15$.

A simple checkup of the simulation algorithm is to compare the results obtained for $\alpha=0$, that is, no coupling between elastic and magnetic degrees of freedom with the results obtained from a Metropolis simulation of an Ising model on a fixed lattice. Figure 1 shows such a comparison for the specific heat of a $L=16$ square lattice. The orange curve corresponds to the static Ising system. As expected, the decoupled elastic system is simply a sum of the elastic contribution $\left(C_{v}^{\mathrm{el}}=1\right)$ and the magnetic contribution which is identical to the static system, showing the same finite-size broadened peak at the ordering temperature.

\section{RESULTS}

\section{A. Square lattice}

In what follows we will describe the results obtained for ferromagnetic interactions. The antiferromagnetic case can be obtained by the usual mapping $S_{A} \rightarrow-\tilde{S}_{A}, S_{B} \rightarrow \tilde{S}_{B}$, where $A$ and $B$ are the two disjoint sublattices of the square lattice. We find it useful in terms of presenting the results to separate the 


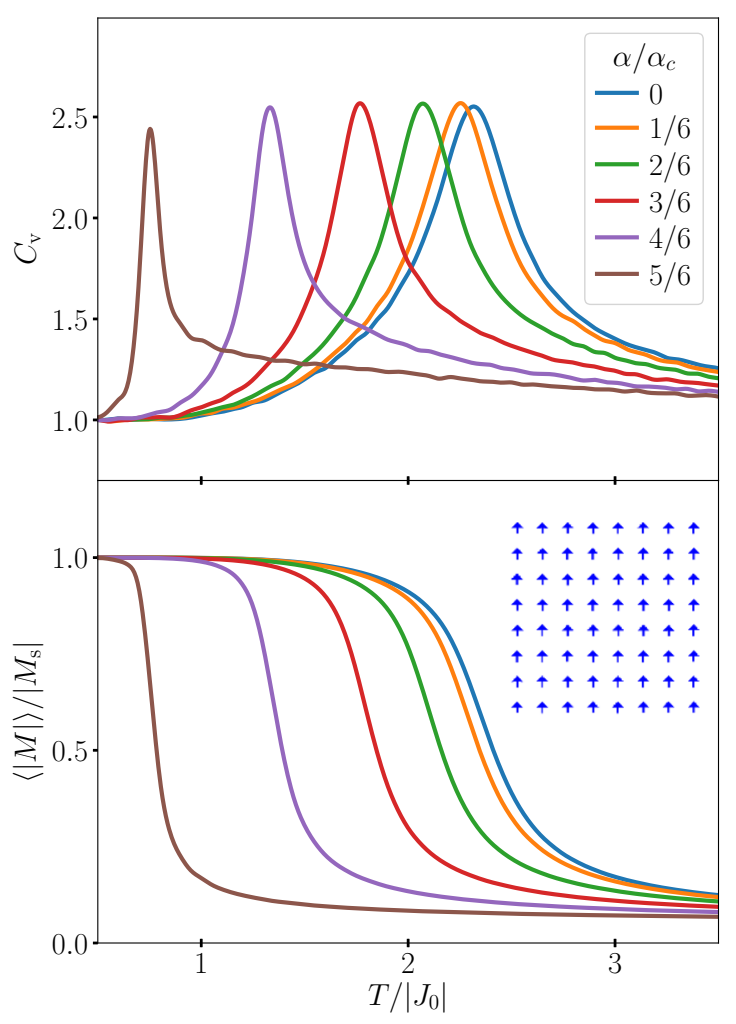

FIG. 2. Specific heat $C_{v}$ (upper panel) and magnetization $M$ (lower panel) as a function of temperature for a series of fixed values of $\alpha$ below $\alpha_{c}$ (see legend). The ferromagnetic transition progressively moves to lower temperatures as $\alpha$ is increased until it eventually vanishes at $\alpha_{c}$. The lower panel shows a snapshot of the ordered state.

discussion for values of $\alpha$ above and below the critical value $\alpha_{c}$ at which the ordering transition vanishes.

\section{1. $\alpha<\alpha_{c}$ : The ferromagnetic transition}

Figure 2 shows the specific heat and magnetization (the order parameter for the FM transition) as a function of temperature as obtained from our simulations for a series of runs with increasing values of the coupling parameter $\alpha$. The data show that the ferromagnetic (FM) transition moves towards lower temperatures as $\alpha$ is increased. As expected, the peak in the specific heat (upper panel) becomes sharper as the critical temperature is reduced, and so does the step towards saturation in the magnetization (lower panel). If $\alpha$ is further increased, the FM transition temperature sinks towards zero at $\alpha_{c}=60$ (for this given value of $K_{\mathrm{e}}$ ). A calculation of $\alpha_{c}$, which becomes clear once the ordered state for high values of $\alpha$ is known, is given in the Appendix.

Figure 3 shows the mean value of the displacement $\langle u\rangle$ for the same range of temperatures and coupling parameters. The curve for $\alpha=0$ follows the expected square root behavior starting at $\langle u\rangle=0$ at $T=0$. The coupled systems follow that same curve at low temperatures, while the system is ordered, but the disordering transition in the magnetic part is accompanied by a sudden increase in $\langle u\rangle$. It is straightforward to calculate from this data the mean value of the pair exchange

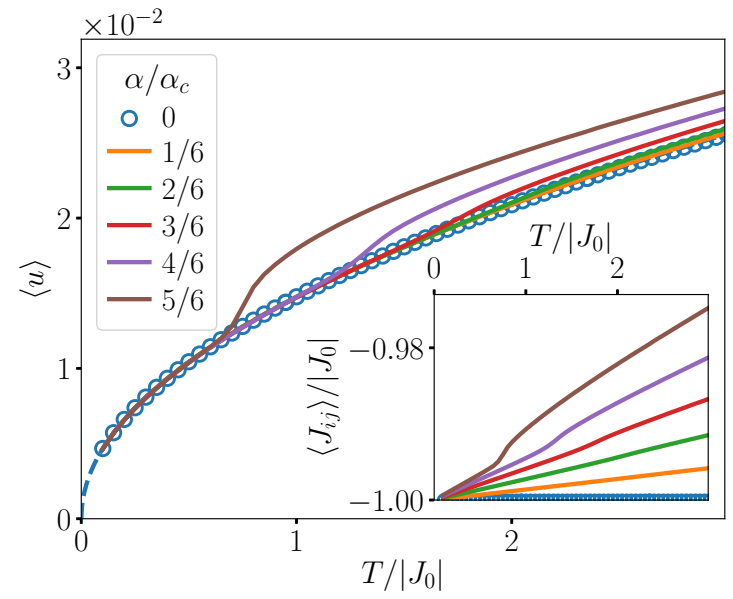

FIG. 3. Mean value of the displacement $\langle u\rangle$ as a function of temperature for different $\alpha$ below $\alpha_{c}$. In the absence of any coupling ( $\alpha=0$ ) the curve follows the expected $\sqrt{T}$ behavior (dotted line). For coupled systems the displacement follows the same curve at low temperatures and jumps up at the magnetic transition and follows a $\sqrt{T}$ dependence with an increasingly higher prefactor (see text). The inset shows $\left\langle J_{i j}\right\rangle$ as a function of $T$ for the same values of $\alpha$.

constant $\left\langle J_{i j}\right\rangle$ seen in the inset of the same figure, but it offers little explanation as for the cause of the suppression of order as a function of $\alpha$. The origin of the decrease in $T_{C}$, and eventual disappearance of the FM phase is twofold. One factor is that even if the mean value of $J_{i j} /\left|J_{0}\right|$ is always close to -1 , the dispersion increases rapidly as a function of temperature. For small $\alpha$, the tail of the distribution with weaker $J_{i j}$ values dominates the transition temperature. For higher values of $\alpha$, but still below $\alpha_{c}$, another mechanism becomes important: the stabilization of magnetization domains. As usual in any Ising transition, the systems start splitting into domains of opposite magnetization, but the unusual mechanism in this case is that the antiferromagnetic walls between domains are accompanied by distortions that change the sign of the exchange constant and thus render them stable. This mechanism favors the existence of domains of opposite magnetization of different sizes and thus conspires against the FM order. To ascertain the existence of these two mechanisms beyond the mere inspection of snapshots, we constructed a histogram of $J_{i j}$ as a function of temperature. The upper panel of Fig. 4 shows such a histogram for $\alpha / \alpha_{c}=5 / 6$ using a data window in $J_{i j} /\left|J_{0}\right|$ between -20 and 20 , with a binning of 0.002 , and collecting data over $P=10^{7} \mathrm{MCS}$. The distribution resembles a Gaussian centered around $J_{i j} /\left|J_{0}\right|=-1$ that increases its half-maximum width as the temperature is increased. However, a closer inspection reveals that the distribution is slightly skewed towards positive $J_{i j}$. A quantitative way of seeing this is by comparing the value of $J_{i j}$ at the maximum with $\left\langle J_{i j}\right\rangle$, which should coincide for a symmetric distribution. This is shown in Fig. 5 for $\alpha / \alpha_{c}=5 / 6$. Below the FM transition, $T_{c}(5 / 6) /\left|J_{0}\right|=0.75$, both the maximum and the mean value coincide, but at $T_{c}$ there is a jump after which the maximum lies at a considerably lower value than the mean. This is evidence of the stabilization of positive values of $J_{i j}$ around the domain borders. This type of mechanism is particular to 

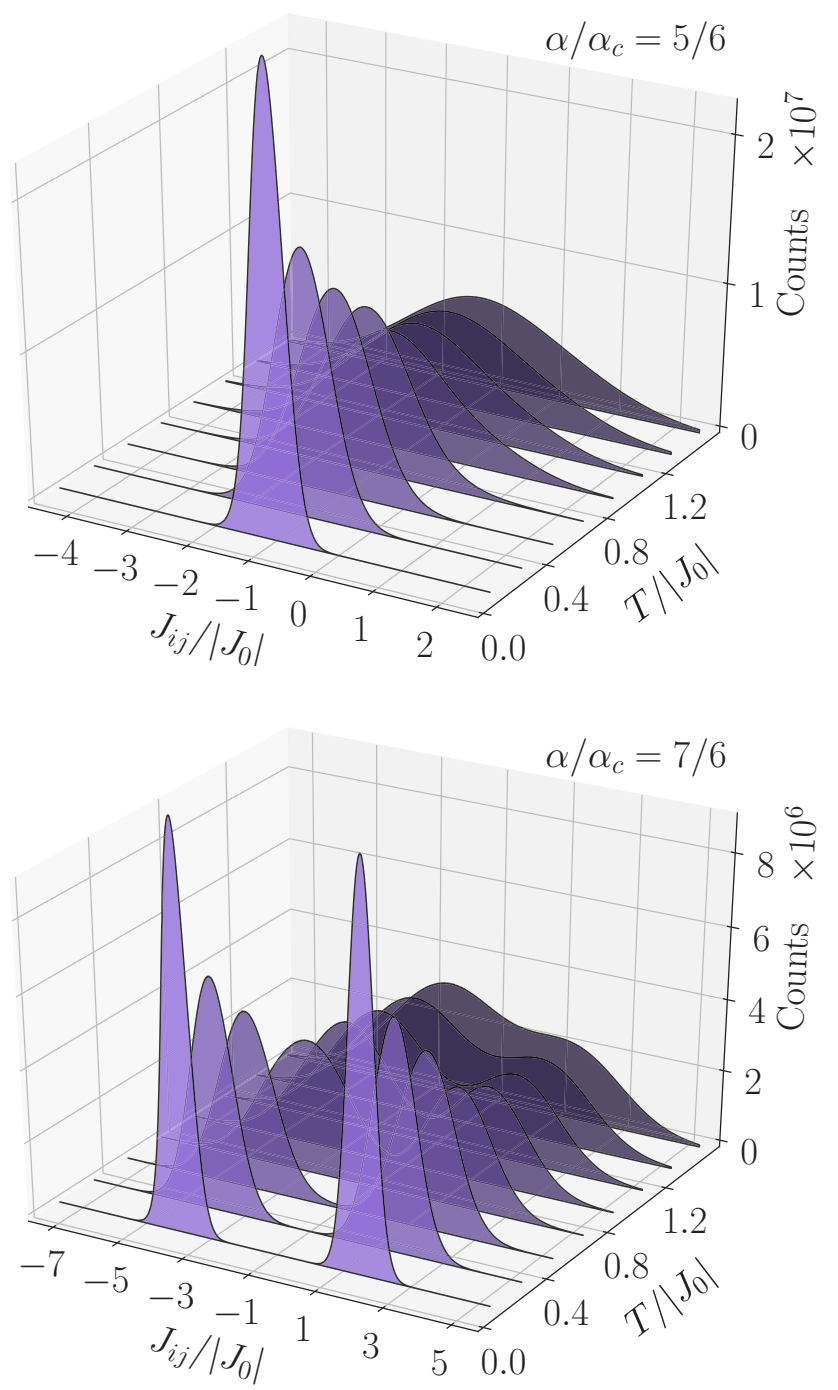

FIG. 4. Histograms for the pair exchange constants $J_{i j}$ at different temperatures. In the upper panel for $\alpha<\alpha_{c}$, and in the lower panel for $\alpha>\alpha_{c}$. Below $\alpha_{c}$ the distribution resembles a Gaussian centered around -1 , but closer inspection shows it is skewed to the right at temperatures close to $T_{c} /\left|J_{0}\right|=0.75$ (see text for details). Above $\alpha_{c}$ it is a bimodal distribution with two clearly defined FM and AFM peaks that merge as the temperature is raised.

the Ising case and should be absent in a Heisenberg system. Indeed, numerical studies of a coupled spin-lattice system with Heisenberg-like spins show that in this case the transition is only marginally affected by the coupling to vibrations [24].

\section{2. $\alpha>\alpha_{c}$ : The checkerboard transition}

If the coupling parameter $\alpha$ continues to be increased, a new ordered state develops at low temperatures. The upper panel of Fig. 6 shows the specific heat above $\alpha_{c}$. As seen in the figure, there is a sharp peak in the specific heat for $\alpha>\alpha_{c}$ that increases in temperature as $\alpha$ is increased. A snapshot of the ordered state that is found at low temperatures in this case is shown in the upper part of Fig. 7. This is a zero-magnetization state where the lattice breaks into clusters of four spins with equal orientation, ordered antiferromagnetically with respect

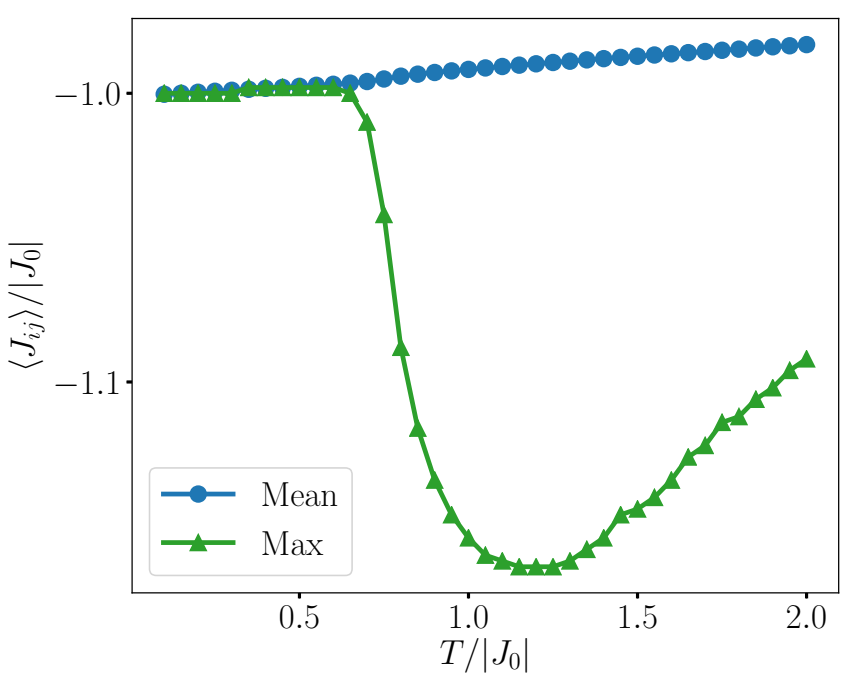

FIG. 5. The value of $J_{i j}$ at the maximum (green triangles) compared with the mean value $\left\langle J_{i j}\right\rangle$ (blue circles) for $\alpha / \alpha_{c}=5 / 6$. These should coincide for a symmetric distribution, instead, there are seen to diverge above $T_{c}$. This is the evidence of the stabilization of positive values of $J_{i j}$ around the domain borders (see text).

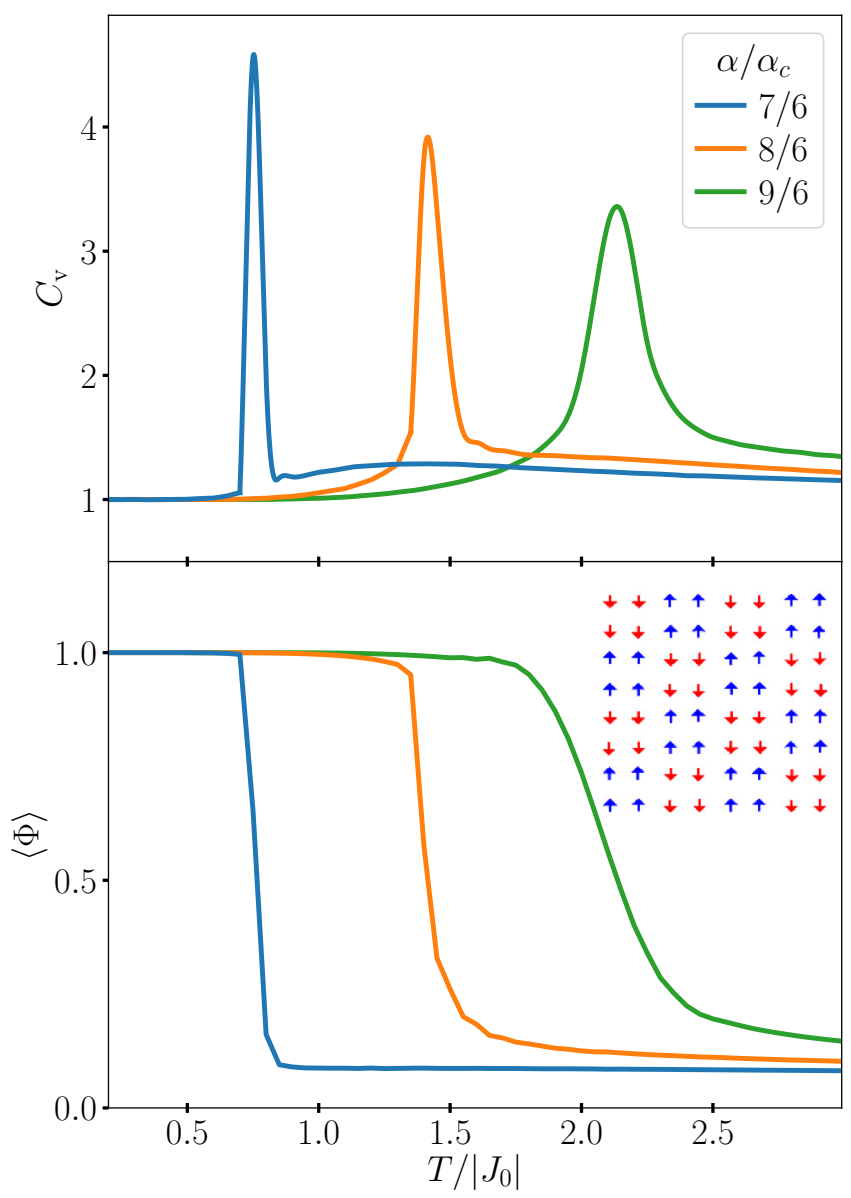

FIG. 6. Specific heat $C_{v}$ (upper panel) and CB order parameter $\Phi$ (lower panel) as a function of temperature for a series of fixed values of $\alpha$ above $\alpha_{c}$ (see legend). Increasing the value of $\alpha$ above $\alpha_{c}$ helps stabilizing the $\mathrm{CB}$ phase at progressively higher temperatures. The lower panel shows a snapshot of the ordered state. 


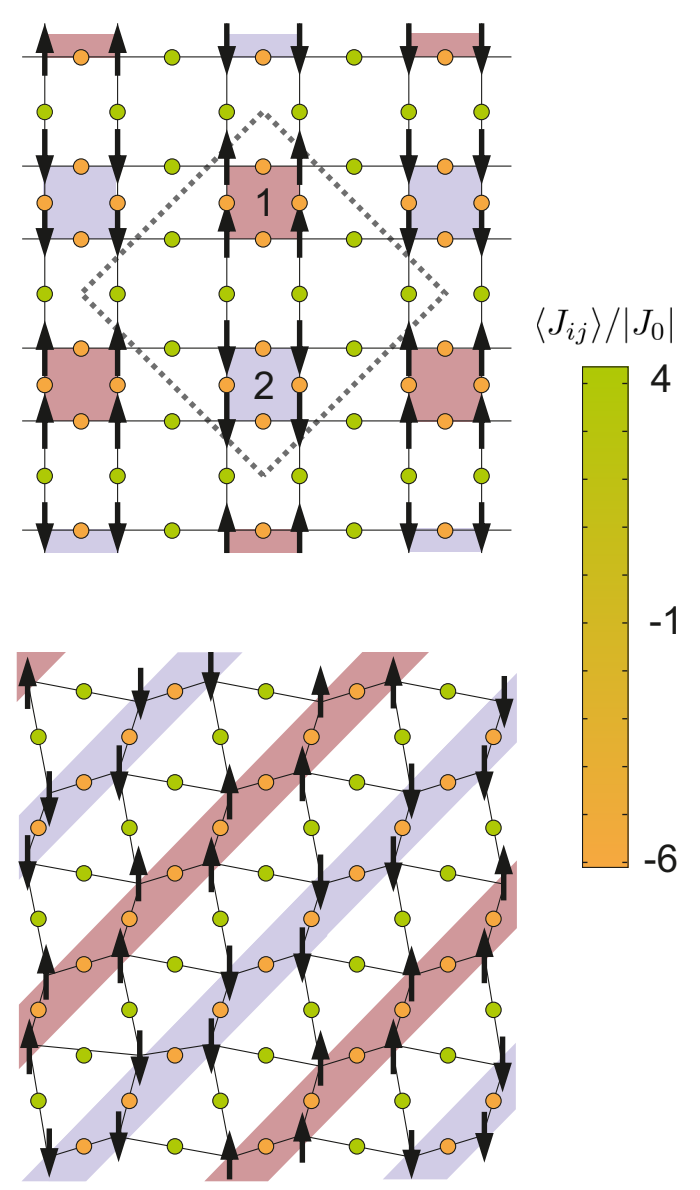

FIG. 7. Two snapshots of configurations for $\alpha>\alpha_{c}$. The upper panel shows an example of one of the possible checkerboard states. Here the lattice dimerizes into square clusters of equal spin orientation aligned antiferromagnetically with respect to each other. The dotted line shows the unit cell. The colored circles correspond to the values of the $\left\langle J_{i j}\right\rangle$ in the bond, and are colored according to the scale on the right. The lower panel shows a stripped phase. This is a low temperature excitation of the CB phase that takes place for values of $\alpha$ close to $\alpha_{c}$. For visual clarity, in both cases the distortions have been exaggerated tenfold.

to each other. We have shaded the clusters in red and blue to emphasise the checkerboard nature of this state. The pair exchange interactions $\left\langle J_{i j}\right\rangle$ are shown as circles in the midpoint between bonds, colored according to the scale shown on the right. The distortions are exaggerated tenfold in the picture for visual clarity.

This state (which we will call CB for short) is a sort of dimerization in two dimensions: the spins in the clusters are closer to each other (thus enhancing ferromagnetic interactions) and further apart from their neighbors in the other cluster (thus turning this interaction antiferromagnetic). It is straightforward to notice that the $J_{i j}$ show a bimodal FMAFM distribution, which is readily seen in the histograms for $\alpha>\alpha_{c}$. An example of these is shown in the lower panel of Fig. 4 , for $\alpha / \alpha_{c}=7 / 6$. Below the transition temperature $\left[T_{c}(7 / 6) \approx 0.7\right]$ there are two separate peaks that evolve into two sharply defined identical peaks at low temperatures at $-4\left|J_{0}\right|$ and $2\left|J_{0}\right|$ (averaging $-\left|J_{0}\right|$ ).

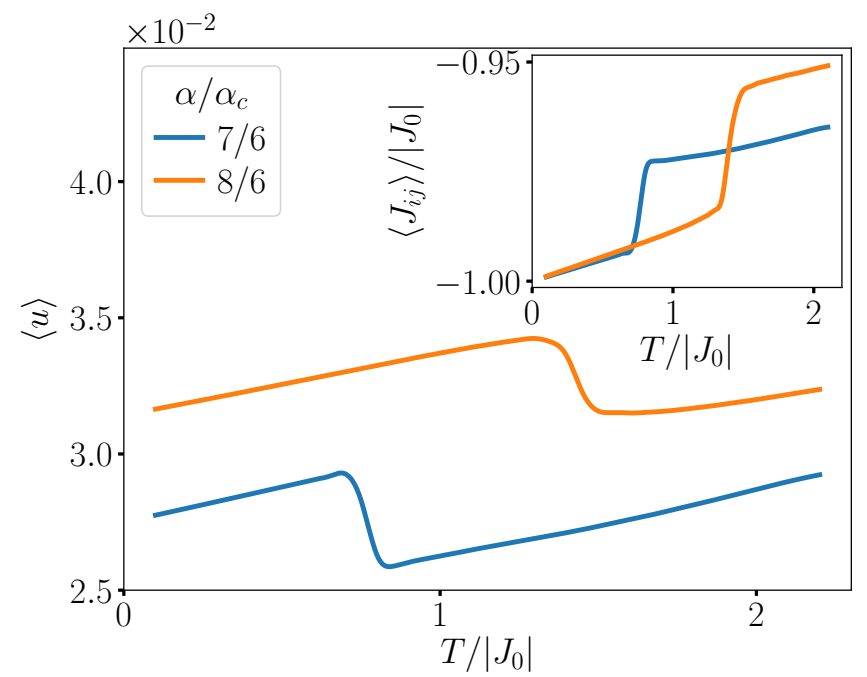

FIG. 8. Mean value of the displacement $\langle u\rangle$ as a function of temperature for different $\alpha$ above $\alpha_{c}$. The transition into the CB phase is marked by a jump in $\langle u\rangle$ which then intercepts $T=0$ at a nonzero value. This is the consequence of a structural transition simultaneous with the magnetic one. The inset shows the mean value of the exchange constant.

To characterize this transition it is useful to calculate an order parameter. We use a unit-cell like the one shown in Fig. 7. We define an index $j$ that runs over all squares in the lattice such that it counts as odd and even the squares marked with 1 and 2, respectively, in the picture, and an index $a$ that runs over the spins in the squares. There are four possible degeneracies of the ground state (plus time reversal), corresponding to where the colored squares are set in the unit cell. We then define an order parameter $\Phi$ that is the sum over the four possibilities $\Phi=1 / N \sum_{m=0}^{4}(-1)^{m}\left|\Phi_{m}\right|$ where

$$
\Phi_{m}=\sum_{j=1}^{N / 4} \sum_{a=1}^{4}(-1)^{j} e^{i \phi_{a}^{m}} \sigma_{a}^{j}
$$

Here $\sigma_{a}^{j}$ are Ising-spin variables that can take the values $\pm 1, N$ is the total number of spins, and the $\phi_{a}^{m}$ are the phase factors for the spin that take into account the four possible degeneracies: $\phi^{1}=\pi(0,0,0,0), \phi^{2}=\pi(1,0,1,0)$, $\phi^{3}=\pi(1,1,0,0), \phi^{4}=\pi(1,0,0,1)$.

The lower panel of Fig. 6 shows the evolution of the order parameter $\phi$ as a function of temperature for different fixed values of $\alpha$ (indicated in the figure). As expected, there is a jump in $\phi$ that coincides with the peak in the specific heat. The jump is sharp for $\alpha$ close to $\alpha_{c}$ and softens as $\alpha$ increases. An inspection of the mean value of the displacement $\langle u\rangle$, Fig. 8 shows that the magnetic ordering corresponds with a jump in $\langle u\rangle$, i.e., there is a simultaneous magnetic and structural transition. This jump in $\langle u\rangle$ in turn relates to the separation of the peaks in the histogram of $J_{i j}$ that we have discussed earlier. Figure 8 also shows that $\langle u\rangle$ remains nonzero as $T \rightarrow$ 0 above $\alpha_{c}$. It is straightforward to calculate $\langle u(T=0)\rangle$ as the minimum from the two possible ground states (FM and $\mathrm{CB}$ ). 


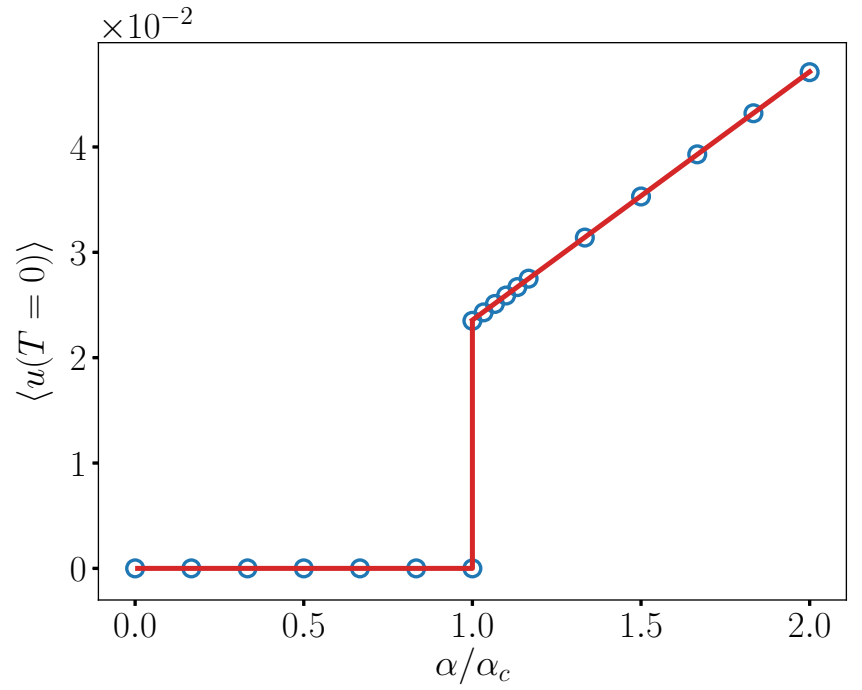

FIG. 9. Distortion at $T=0$ as a function of the coupling parameter $\alpha$. The blue circles correspond to the values obtained from the simulation, and the red line to those predicted by Eq. (4). The sharp step at $\alpha_{c}=60$ marks the structural transition.

The expression, calculated in the Appendix, is simply

$$
u_{\min }(\alpha)= \begin{cases}0 & \text { if } \alpha \leqslant \alpha_{c}, \\ \frac{\sqrt{8}}{K_{\mathrm{el}}} \alpha & \text { if } \alpha>\alpha_{c} .\end{cases}
$$

This is shown as a red line in Fig. 9 together with the values obtained from the simulations (open circles). As it can be seen there, there is a sharp step in $\langle u(T=0)\rangle$ at $\alpha_{c}$ corresponding to a structural transition that lowers the lattice symmetry.

When $\alpha$ is close to $\alpha_{c}$ the distortions are still small and there are states with long-range order that have energies comparable with the ground state. It is very frequent that for these values of $\alpha$ the simulated system will remain at a local minima. One such possible state is pictured in the bottom part of Fig. 7. This is still a dimerized state, with pairs of equally pointing spins ordered antiferromagnetically, but it corresponds to a shift in the phase (by $a$ ) in consequent horizontal rows. In this state the $J_{i j}$ order in stripes, and so do the spins (shaded red and blue). In all other respects it shares the characteristics of the ground state (bimodal distribution, jump in the distortion, etc.) If the distortion is small, this costs very little energy, but it becomes progressively disfavored as $\langle u\rangle$ increases. The snapshot corresponds to a small distortion configuration, but, as we mentioned before, the distortion in the figure has been multiplied by an order of magnitude to make it apparent.

Similar phases are known to be brought about by coupling to lattice distortions in a different context. This is the case of the phonon-induced phases found in the Holstein-Hubbard model [25-29]. This is model of a correlated electron system where electron-phonon interactions with Einstein phonons are considered in addition to electron-electron interactions. Contrary to our case, this model treats phonons quantum mechanically and has a coupling to elastic degrees of freedom that is odd in nature, since it was originally conceived for a molecular crystal. For large values of the coupling strength,

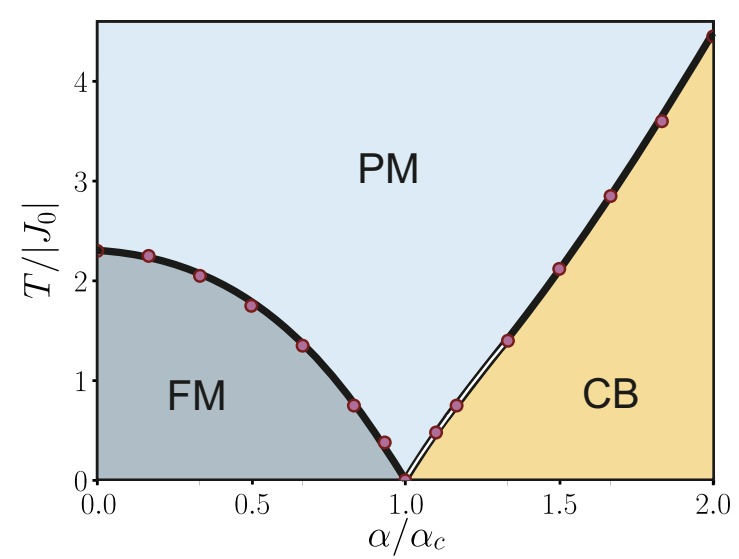

FIG. 10. The $T-\alpha$ phase diagram for the FM Ising model on a square lattice obtained from the simulations. The transitions separate a high temperature paramagnet (PM) from the two ground states: the ferromagnet (FM) and the checkerboard (CB). The single line marks a second-order phase transition while the double line marks first order. The circles, taken from the position of the peak in the specific heat, show some of the data points used to construct the phase diagram.

a bipolaronic insulator emerges that is reminiscent of the $\mathrm{CB}$ phase found here.

\section{3. $T$ - $\alpha$ phase diagram}

The $T-\alpha$ phase diagram of this system is a sort of summary of the results discussed up to this point. Figure 10 shows the $T-\alpha$ phase diagram as obtained from the simulations. The circles in the figure correspond to the position of the peak in the specific heat.

In the absence of any coupling $(\alpha=0)$ we find the Ising transition from the high temperature paramagnet (PM) into the ferromagnetically ordered state (FM). This second-order transition decreases in temperature as $\alpha$ is increased until it sinks to $T=0$ at $\alpha_{c}$. When the coupling is increased beyond this point a new ground state emerges, the checkerboard (CB), which is a combination of antiferromagnetically ordered ferromagnetic clusters. The $\mathrm{CB}$ transition is simultaneous with a structural transition that decreases the symmetry of the lattice.

As we mentioned, the transition below $\alpha_{c}$ is the expected second-order transition in the Ising universality class. This is not the case above $\alpha_{c}$. The simultaneous occurrence of the magnetic and structural transitions alters the nature of the transition which seems to be first order in the range $1.0 \leqslant$ $\alpha / \alpha_{c} \lesssim 1.3$, as determined from the behavior of the Binder cumulant (not shown) [30,31]. Some properties show hysteresis in this region when sweeping the temperature up and down. The exact mechanism that determines the range of existence of this first-order region is matter of future investigation.

\section{B. Kagomé lattice}

We have applied this same simulation algorithm and data analysis to the case of the Ising model on the Kagomé lattice. For the FM case the results follow closely those of the square lattice. In the AFM case, which is frustrated, the systems remain disordered up to a critical value of $\alpha$ above which the 


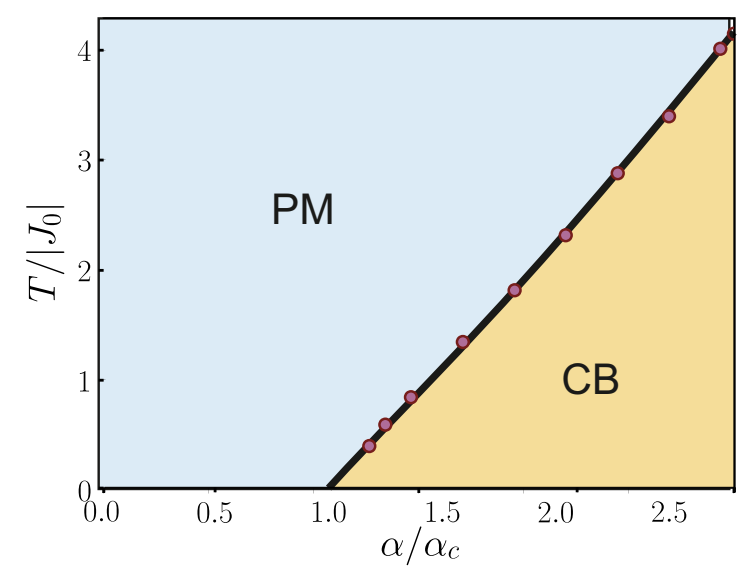

FIG. 11. The $T-\alpha$ phase diagram for the AFM Ising model on the Kagomé lattice obtained from the simulations. For low values of $\alpha$ there is no long range order at any temperature. Above $\alpha_{c}$ the coupling to the lattice degrees of freedom results in a lifting of the frustration into a checkerboard (CB) phase at low temperatures. The circles, taken from the position of the peak in the specific heat, show some of the data points used to construct the phase diagram.

frustration is lifted through a simultaneous structural and magnetic transition into the Kagomé CB phase (see Fig. 11). The Kagomé CB, pictured in Fig. 12, can also be understood as a dimerization along the three different axes of the lattice (pictured as ovals of different shades). However, the distribution of the $J_{i j}$ is slightly different, since it is now trimodal, with two different FM exchange constants corresponding to triangular and hexagonal FM clusters, pictured in Fig. 12 in red and blue, respectively, which are oriented antiferromagnetically with respect to each other. This is also a zero magnetization state, since the number of triangles is twice the number of hexagons.

\section{CONCLUSIONS}

In this work we have studied the simple classical twodimensional Ising model on a square and on a Kagomé lattice with Einstein distortions. We have performed Monte Carlo simulations of the linearly coupled system taking into account simultaneously both degrees of freedom. In both the unfrustrated square lattice and the frustrated Kagomé lattice we find that when the coupling is increased above a critical value the system has a structural transition-a dimerization along the lattice axes. This occurs simultaneously with magnetic ordering into a clustered state with zero magnetization, composed of squares in the square lattice, and triangular and hexagonal FM clusters in the Kagomé case. These clusters correspond to the appearance of a bimodal distribution of exchange constants in the square lattice, one intracluster FM and one intercluster AFM, and a trimodal distribution in the Kagomé case: two FM interactions (intratriangles and intrahexagons) and an AFM interaction intercluster. In the unfrustrated case we show that the coupling to the elastic degrees of freedom gradually weakens the transition, through a mechanism whereby domain formation is gradually stabilized by distortions. In the square lattice we identified low-energy
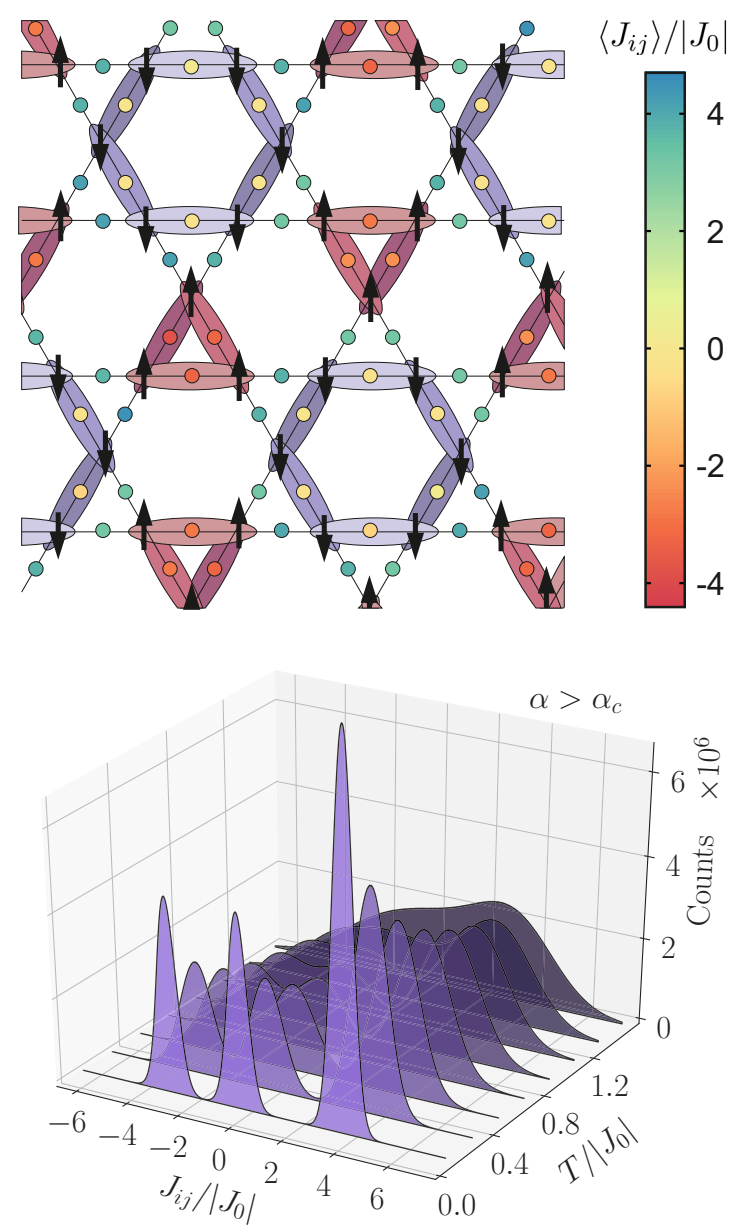

FIG. 12. Upper panel: Snapshot of the Kagomé CB. The ovals of different shades mark the dimerization along the three different directions. The distribution of $J_{i j}$ is in this case trimodal, with one AFM peak and two FM constants corresponding to the red triangular and the blue hexagonal clusters. $\left\langle J_{i j}\right\rangle$ is marked by colored circles located midpoint between the spins, colored according to the scale on the right. Lower panel: Histogram of the Kagomé CB state obtained from a system of $N=216$ spins showing a trimodal distribution with two peaks at FM couplings and one AFM.

excitations consisting of stripes of zigzagging spins. The analysis of the phase diagram shows that the transition into the ordered state is not always second order, but further work is needed to identify the exact boundaries and the mechanisms that are responsible for this.

The main aim of this work was to study one of the simplest possible models with magnetoelastic coupling, and hence the choice of the Ising model on two dimensions with linear coupling between $J$ and $u$. It can still be questioned whether such a simple model would have any remit of applicability. Detailed descriptions of the dependence of $J$ with $u$ in real materials are scarce. References [32,33] provide a careful discussion of the dependence of the magnetic coupling constants of the compound $\mathrm{CuGeO}_{3}$ with respect to lattice distortions. The main magnetic interaction in this case is given by superexchange paths, but if one makes a simple geometrical model to translate variations in the angle of the mediated pathway into relative displacement between the two 


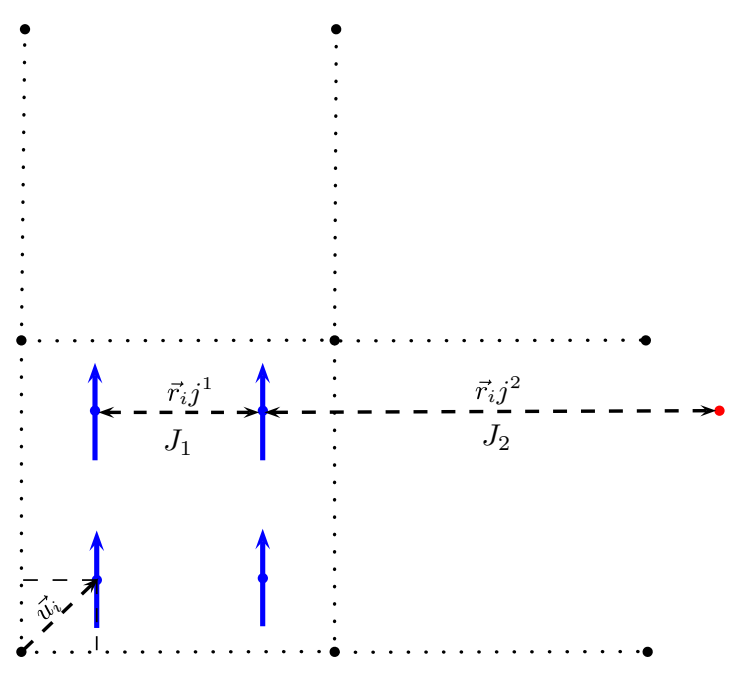

FIG. 13. Schematic view of the CB cell used for the calculation of the energy.

magnetic sites, one finds that for the parameters of $\mathrm{CuGeO}_{3}$ displacements in $u_{i j}$ of the order of $3 \%$ are well described by a linear dependence of $J(u)$ with a coupling constant that varies from 10 to 90 depending on the value chosen for the undistorted angle, i.e., for $\alpha / \alpha_{c}$ between 0.16 and 1.5 in the simple Ising model presented here.

\section{ACKNOWLEDGMENTS}

We are grateful to R. A. Borzi for discussions and a careful read of the manuscript. This work was supported by CONICET (Argentina), and ANPCYT (Argentina) via Grant PICT-2013-2004.

\section{APPENDIX: CALCULATION OF THE GROUND STATE AT $T=0$ FOR THE SQUARE LATTICE}

At $T=0$ it is straightforward to calculate the critical value of the coupling parameter $\alpha_{c}$ above which the CB phase is energetically favorable over the FM phase.

We start by calculating the energy in the CB configuration. Figure 13 shows a schematic view of the cell used for this calculation.
Taking the assumption that at $T=0 \vec{u}_{i}$ has identical projections along $x$ and $y$ and one gets

$$
r_{i j}^{1,2}=1 \mp 2 \frac{u}{\sqrt{2}},
$$

where $u \equiv u_{i}=\left|\vec{u}_{i}\right|$. The value of the two exchange constants is then given by

$$
J_{1,2}=J_{0}\left[1-\alpha\left(1 \mp 2 \frac{u}{\sqrt{2}}-1\right)\right]=J_{0}(1 \pm \alpha \sqrt{2} u)
$$

where we have used that $S_{i} S_{j}= \pm 1$ for 1 and 2 , respectively.

Thus, the energy per spin of the given unit cell (Fig. 13), is given by

$$
\begin{aligned}
{ }_{\varepsilon}^{\mathrm{CB}} & =\frac{E^{\mathrm{CB}}}{N}=\frac{4 J_{1}-4 J_{2}+4\left|J_{0}\right| \frac{K_{\mathrm{el}}}{2} u^{2}}{4} \\
& =2 \sqrt{2} J_{0} \alpha u+\left|J_{0}\right| \frac{K_{\mathrm{el}}}{2} u^{2} .
\end{aligned}
$$

Minimizing the energy with respect to the displacement $u$ one obtains the displacement at minimal energy $u_{\min }=\sqrt{8} \frac{\alpha}{K_{\mathrm{el}}}$, which in turn gives the energy

$$
\varepsilon_{\min }^{\mathrm{CB}} \equiv \varepsilon^{\mathrm{CB}}\left(u_{\min }\right)=-4 \frac{\alpha^{2}}{K_{\mathrm{el}}}\left|J_{0}\right| .
$$

On the other hand, the energy of the FM phase is trivially

$$
\varepsilon_{\mathrm{min}}^{\mathrm{FM}}=\frac{E_{\mathrm{min}}^{\mathrm{FM}}}{N}=-2\left|J_{0}\right| \text {. }
$$

By equating Eqs. (A5) and (A6) one obtains

$$
\alpha_{c}=\sqrt{\frac{K_{\mathrm{el}}}{2}}
$$

which gives $\alpha_{c}=60$ for the parameters used in this work. This is in good agreement with the value determined by the MC simulations.

It is probably worth noticing that, were the sign of $S_{i} S_{j}$ in $J_{2}$ not be subject to inversion, then $J_{1}+J_{2}=2 J_{0}$, which means that with a linear $J_{i j}$ no deformation would be stable since there would no longer be any gain in the magnetic part of the energy.
[1] P. Pincus, Solid State Commun. 9, 1971 (1971).

[2] M. Hase, I. Terasaki, and K. Uchinokura, Phys. Rev. Lett. 70, 3651 (1993).

[3] L. Gu, B. Chakraborty, P. L. Garrido, M. Phani, and J. L. Lebowitz, Phys. Rev. B 53, 11985 (1996).

[4] O. Tchernyshyov, R. Moessner, and S. L. Sondhi, Phys. Rev. Lett. 88, 067203 (2002).

[5] O. Tchernyshyov, R. Moessner, and S. L. Sondhi, Phys. Rev. B 66, 064403 (2002).

[6] K. Penc, N. Shannon, and H. Shiba, Phys. Rev. Lett. 93, 197203 (2004).

[7] C. Weber, F. Becca, and F. Mila, Phys. Rev. B 72, 024449 (2005).
[8] D. L. Bergman, R. Shindou, G. A. Fiete, and L. Balents, Phys Rev. B 74, 134409 (2006).

[9] F. Wang and A. Vishwanath, Phys. Rev. Lett. 100, 077201 (2008).

[10] Y. Shokef, A. Souslov, and T. C. Lubensky, Proc. Natl. Acad. Sci. U.S.A. 108, 11804 (2011).

[11] K. Uchinokura, J. Phys.: Condens. Matter 14, R195 (2002).

[12] H. Ueda, H. A. Katori, H. Mitamura, T. Goto, and H. Takagi, Phys. Rev. Lett. 94, 047202 (2005).

[13] S. Kimura, M. Hagiwara, H. Ueda, Y. Narumi, K. Kindo, H. Yashiro, T. Kashiwagi, and H. Takagi, Phys. Rev. Lett. 97, 257202 (2006). 
[14] M. Matsuda, H. Ueda, A. Kikkawa, Y. Tanaka, K. Katsumata, Y. Narumi, T. Inami, Y. Ueda, and S.-H. Lee, Nat. Phys. 3, 397 (2007).

[15] Y. Tanaka, Y. Narumi, N. Terada, K. Katsumata, H. Ueda, U. Staub, K. Kindo, T. Fukui, T. Yamamoto, R. Kammuri et al., J. Phys. Soc. Jpn. 76, 043708 (2007).

[16] R. Valdés Aguilar, A. B. Sushkov, Y. J. Choi, S.-W. Cheong, and H. D. Drew, Phys. Rev. B 77, 092412 (2008).

[17] S. Zherlitsyn, O. Chiatti, A. Sytcheva, J. Wosnitza, S. Bhattacharjee, R. Moessner, M. Zhitomirsky, P. Lemmens, V. Tsurkan, and A. Loidl, J. Low Temp. Phys. 159, 134 (2010).

[18] V. Tsurkan, S. Zherlitsyn, S. Yasin, V. Felea, Y. Skourski, J. Deisenhofer, H.-A. K. von Nidda, J. Wosnitza, and A. Loidl, Phys. Rev. Lett. 110, 115502 (2013).

[19] Y. Yamashita and K. Ueda, Phys. Rev. Lett. 85, 4960 (2000).

[20] F. Becca and F. Mila, Phys. Rev. Lett. 89, 037204 (2002).

[21] C. Jia and J. H. Han, Physica B 378, 884 (2006).
[22] See Supplemental Material at http://link.aps.org/supplemental/ 10.1103/PhysRevB.99.144421 for a convergence test of the quantities measured in the simulations.

[23] X. Zheng and J. Earnshaw, Europhys. Lett. 41, 635 (1998).

[24] D. Perera, T. Vogel, and D. P. Landau, Phys. Rev. E 94, 043308 (2016).

[25] M. Capone, G. Sangiovanni, C. Castellani, C. Di Castro, and M. Grilli, Phys. Rev. Lett. 92, 106401 (2004).

[26] P. Werner and A. J. Millis, Phys. Rev. Lett. 99, 146404 (2007).

[27] J. Bauer and A. C. Hewson, Phys. Rev. B 81, 235113 (2010).

[28] Y. Murakami, P. Werner, N. Tsuji, and H. Aoki, Phys. Rev. B 88, 125126 (2013).

[29] P. Werner and M. Eckstein, Europhys. Lett. 109, 37002 (2015).

[30] K. Binder, Phys. Rev. Lett. 47, 693 (1981).

[31] K. Binder, Z. Phys. B Condens. Matter 43, 119 (1981).

[32] M. Braden, G. Wilkendorf, J. Lorenzana, M. Aïn, G. J. McIntyre, M. Behruzi, G. Heger, G. Dhalenne, and A. Revcolevschi, Phys. Rev. B 54, 1105 (1996).

[33] R. Werner, C. Gros, and M. Braden, Phys. Rev. B 59, 14356 (1999). 